\title{
CORRECTION
}

\section{Correction to: The tracing of trade}

\section{Exploring the patterns of olive oil production and distribution from Roman Baetica}

\author{
María Coto-Sarmiento ${ }^{1}\left[\right.$ [ Xavier Rubio-Campillo ${ }^{2,3}$ \\ Published online: 21 November 2021 \\ ๑) Springer-Verlag GmbH Germany, part of Springer Nature 2021
}

Correction to: Archaeological and Anthropological Sciences (2021) 13: 70 https://doi.org/10.1007/s12520-021-01305-4

In this article the graphics relating to Figs. 9 and 10 are the same; the figure(s) should have appeared as shown below.

The original article has been corrected.

The original article can be found online at https://doi. org/10.1007/s12520-021-01305-4.

María Coto-Sarmiento

mcotsar@gmail.com

1 Department of Prehistory and Archaeology, Universitat de Barcelona, Montalegre 6-8, 08001, Barcelona, Spain

2 DIDPATRI, Universitat de Barcelona, Passeig de la Vall d'Hebrón, 171, Barcelona, Spain

3 School of History, Classic, Archaeology, William Robertson Wing, University of Edinburgh, Old Medical School, Room OOM.33, Teviot Place, Edinburgh, UK 
Fig. 9 Dendrogram displaying hierarchical clustering for Britannia sites based on Morisita-Horn metrics. Site names are colour-coded by typology: military sites (red), civilian sites (green) and unspecified (grey)
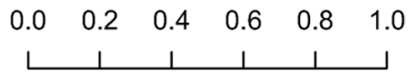

Tayside

South Shields

Old Kilpatrick

Gloucester

Fishbourne

$$
\text { Exeter }
$$

Ilchester

Southwark

Chichester

Watercrook

Canterbury

Winchester

Caerwent

Warrington

Caernarfon

Caerhun

Loughor

Newstead

Bishopbriggs

Falkirk

Cirencester

Carlisle

Leicester
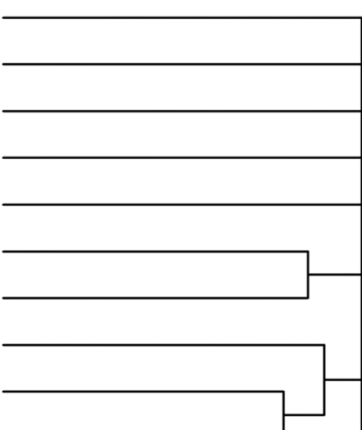

Ribchester

Bar Hill

East Ayrshire
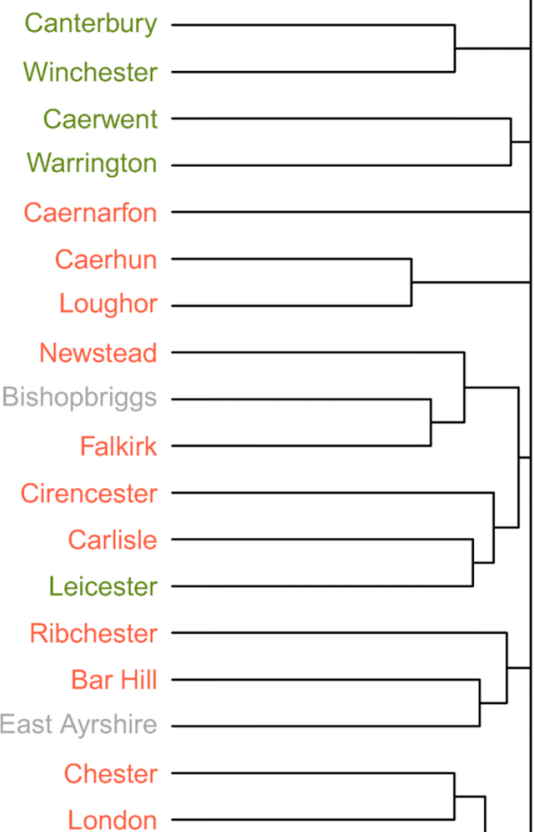

Bardon Mill

St Albans

Caerleon

Corbridge

Silchester

Wroxeter

Colchester

Richborough

Aldborough

Birrens

Newcastle upon Tyne
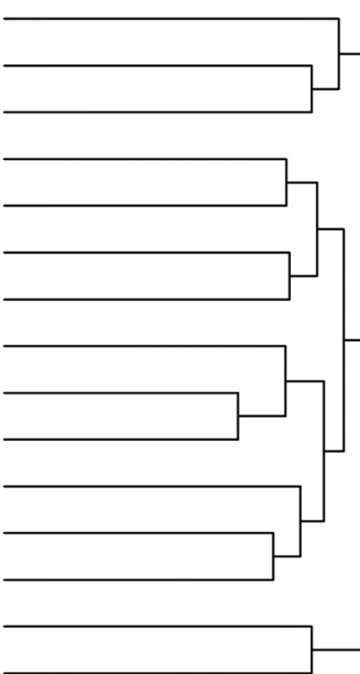

Abernethy

Edinburgh

Chesters

Housesteads

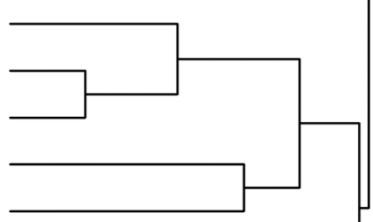

Ilkley

Lincoln

York

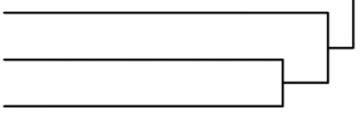


Fig. 10 Dendrogram displaying hierarchical clustering for Germania sites based on Morisita-Horn metrics. Site names are colourcoded by typology: military sites (red), civilian sites (green) and unspecified (grey) $\begin{array}{llllll}0.0 & 0.2 & 0.4 & 0.6 & 0.8 & 1.0\end{array}$
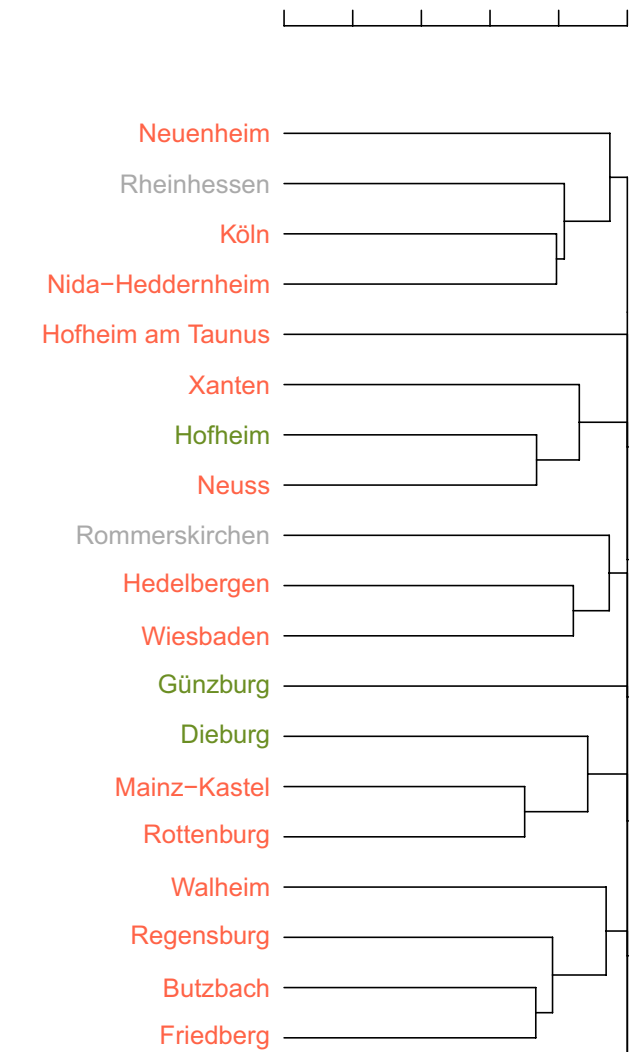

Ladenburg (Lopodunum)

Bad Wimpfen

Sttutgart-Bad Cannstatt

Straubing

Trier

Bad Ems

Hanau

Groß-Gerau

Rottweil

Kempten

Köngen

Bad Cannstatt

Stockstadt am Main
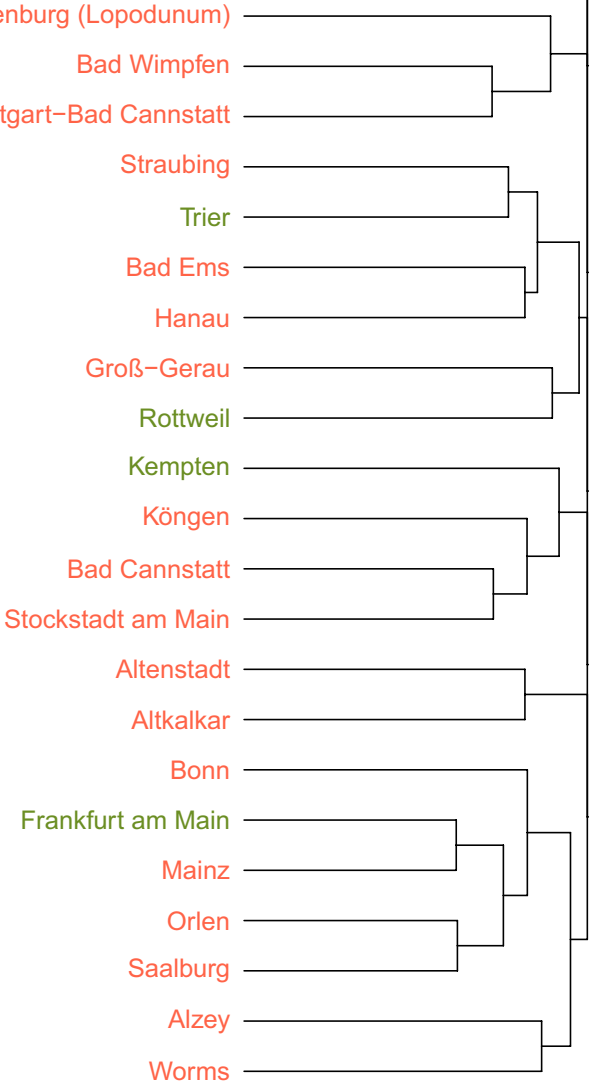